\title{
THE INFLUENCE OF SURFACTANTS ON CASEIN MEMBRANE PREPARATION AND SEPARATION TECHNOLOGIES
}

\author{
Marius Simion ${ }^{1}$, Gabriela Paun ${ }^{2}$, Aurelia Meghea ${ }^{3}$, Jianzhong Ma ${ }^{4}$, Fan Zhang ${ }^{5}$ \\ ${ }^{1}$ INCD ECOIND, Drumul Podu Dambovitei Street, no 71-73, District 6, Bucharest, \\ Romania,email:mariussic@gmail.com \\ 2INCDSB, 296 Splaiul Independenței, District 6, Bucharest, Romania, email: \\ gpaunroman@gmail.com \\ ${ }^{3}$ Faculty of Applied Chemistry and Materials Science Department of Inorganic \\ Chemistry, Polizu Street, District 1, Bucharest, Romania, \\ email:a.meghea@gmail.com \\ ${ }^{4}$ Shaanxi Research Institute of Agricultural Products Processing Technology, Xi'an \\ 710021, Shaanxi Province, PR China,e-mail:miz@sust.edu.cn \\ ${ }^{5}$ College of Resource and Environment, Shaanxi University of Science and \\ Technology, Xi'an 710021, Shaanxi Province, PR China, email: \\ zhangfan21311@126.com
}

\begin{abstract}
The influence of a classic surfactant: palmitoyl-glycylglycine and bola amphiphilic: bis[2butyl(sodium bis-thioacetate) sodium dicarboxylate 1,10 decanediyl ester] upon the casein membrane preparation and separation technologies for removing organic pollutants (dyes) was studied by: UV-VIS and FT/IR-ATR spectroscopy, dynamic light scattering, optical and scanning electron microscopy, contact angle, microbiological tests and the separation rates. The membranes were produced by a casting-solvent evaporation technique. The casein powder was dissolved in a water- acetic acid $(75: 25 \mathrm{v} / \mathrm{v})$ solution with and without plasticizer: glycerol and surfactant (classic or bola), constant continuous stirring for $6-8 \mathrm{hrs}$. at $40^{\circ} \mathrm{C}$, then degassed the solution for $2 \mathrm{hrs}$. The solution was poured and afterwards maintained in the oven at $40-50^{\circ} \mathrm{C}$ for $8-12 \mathrm{hrs}$. Membranes were obtained with different surfactants which influenced the separation properties of membranes.

Ecological membranes are obtained from a biodegradable biopolymer and can be used successfully in removing dyes from wastewaters.
\end{abstract}

Keywords: bola, surfactants, casein, membranes, dyes

\section{Introduction}

Casein is the most used natural polymer (collagen, elastin, fibrin, etc.) due to the great variation of products with controlled reactivity and resorption and the physical, mechanical and biological specific properties. As a natural polymer, casein is a completely biodegradable raw material and has certain forming properties to obtains membranes or films, such as good adhesive force and strong resistance hightemperature, water vapor permeability, polishing. However, casein membranes has less extensibility and water resistance due to its structure [1], which could restrict its practical applications. Chemical modification and blending with other materials like surfactants, or including cross-linkers [2] and polymers [3] for the casein have been attempted to correct its deficiencies. There are two types of membranes, namely simple and composite membranes. In this research simple casein membranes are obtained by a uniform casting of the solution with: casein/water- acetic acid/ glycerol/surfactant 
on a glass plate. Casein membranes were prepared using or not a plasticizer-glycerol and surfactant (classic or bola) such as palmitoyl-glycylglycine and bola amphiphilic: bis[2-butyl(sodium bis-thioacetate) sodium dicarboxylate 1,10 decanediyl ester]. Although the tensile strength and hydrophobic property improved by introducing a surfactant (classic or bola).The surfactants are also involved in the membrane processes, influencing flow through polymeric porous media, cleaning of membranes during the process and after use or modifying the microstructure of the disperse system for separation. In this work the influence of surfactants upon the microporous structure and retention of some pure water soluble dyes: Yellow III (color index 15985) and Orange III (methyl orange), were studied. The surfactant-dye mixed aqueous solutions obtained by varying the dye and surfactant concentration respectively was characterized by UV-VIS spectroscopy, dynamic light scattering, contact angle.

Their environmental applications refer, among others, to the presence of dyes, toxic substances, etc in wastewaters, which became more frequent in the last decades, so adequate solutions for their removal should be found.

\section{Experimental}

For casein membranes obtained the following materials have been used: Casein was purchased from Zhejiang Huatian Co. Ltd; Glycerol and acetic acid from SigmaAldrich. National Institute of Research and Development in Chemical and Petrochemical (ICECHIM from Bucharest, Romania) synthesized [4] and provided:

-palmitoyl-glycylglycine obtained by reaction:

$$
\begin{aligned}
& \mathrm{CH}_{3}-\left(\mathrm{CH}_{2}\right)_{14}-\mathrm{C}_{\mathrm{Cl}}^{\prime \prime}+\mathrm{H}_{2} \mathrm{H}-\mathrm{CH}_{2}-\mathrm{CO}-\mathrm{NH}-\mathrm{CH}_{2}-\mathrm{COOH} \stackrel{\mathrm{NaOH}}{\longrightarrow} \\
& \mathrm{CH}_{3}-\left(\mathrm{CH}_{2}\right)_{14}-\mathrm{C}_{\mathrm{NH}-\mathrm{CH}_{2}-\mathrm{CO}-\mathrm{NH}-\mathrm{CH}_{2}-\mathrm{COOH}}+\mathrm{HCl}
\end{aligned}
$$

-bola amphiphilic: bis [2-butyl (bis-thioacetate, sodium) of sodium dicarboxylate] 1.10 decanediyl ester]:<smiles>NC(=O)CSCC(=O)OCCCCCCCCCCOC(=O)CC(=O)O[Na]</smiles>

Ecological membranes formed were used successfully in removing the dyes from wastewaters: Yellow III (food dye, color index 15985) and Orange III (methyl orange) from Merck.

The experimental techniques used in this study consist in: UV-VIS and FT/IR-ATR spectroscopy, dynamic light scattering, optical and scanning electron microscopy, contact angle, microbiological tests:

- UV-VIS Spectrophotometry: Perkin Elmer Lambda 25 model (200-800nm range, double beam optical system, $4 \mathrm{~nm}$ resolution, scanning rate $240 \mathrm{~nm} / \mathrm{min}$, Abs response type);

- $\quad$ FT/IR-ATR Spectrophotometry: Jasco FT-IR 4200;

- $\quad$ DLS: MALVERN Zetasizer Nano;

- $\quad$ scanning electron microscopy: SEM QUANTA 200 electron microscope; 
- $\quad$ separation device: CELFA MEMBRANE SYSTEME P 28. The separation test was carried out on a $100^{3} \mathrm{~cm}$ cell at $0.3 \mathrm{MPa}$. The useful surface area of the 2, 3 membrane was $12.5 \mathrm{~cm}$. The flow times of $100 \mathrm{~cm}$ of water and surfactants solutions were determined;

- optical microscopy: digital images of the films were taken using Bresser Biolux-AL microscope with video-camera. The magnification of the objective lens used was 10X;

- $\quad$ the contact angles were calculated in terms of the size of drops deposited with microsyringe on the membrane surface making use of a mobile horizontal microscope with ocular micrometer. The membrane samples were attached to a glass slide using two-sided adhesive tape to keep the sample flat. All values reported are the averages of at least ten measurements taken at different locations on the surface and have a maximum error of \pm 20 ;

- microbiological tests: were performed according to European standard using Sabouraud culture medium inoculated with Staphyloccous Aureus

\section{Results and discussion}

A new procedure was proposed for obtaining casein membranes by a casting-solvent evaporation technology, and is presented in figure 1:

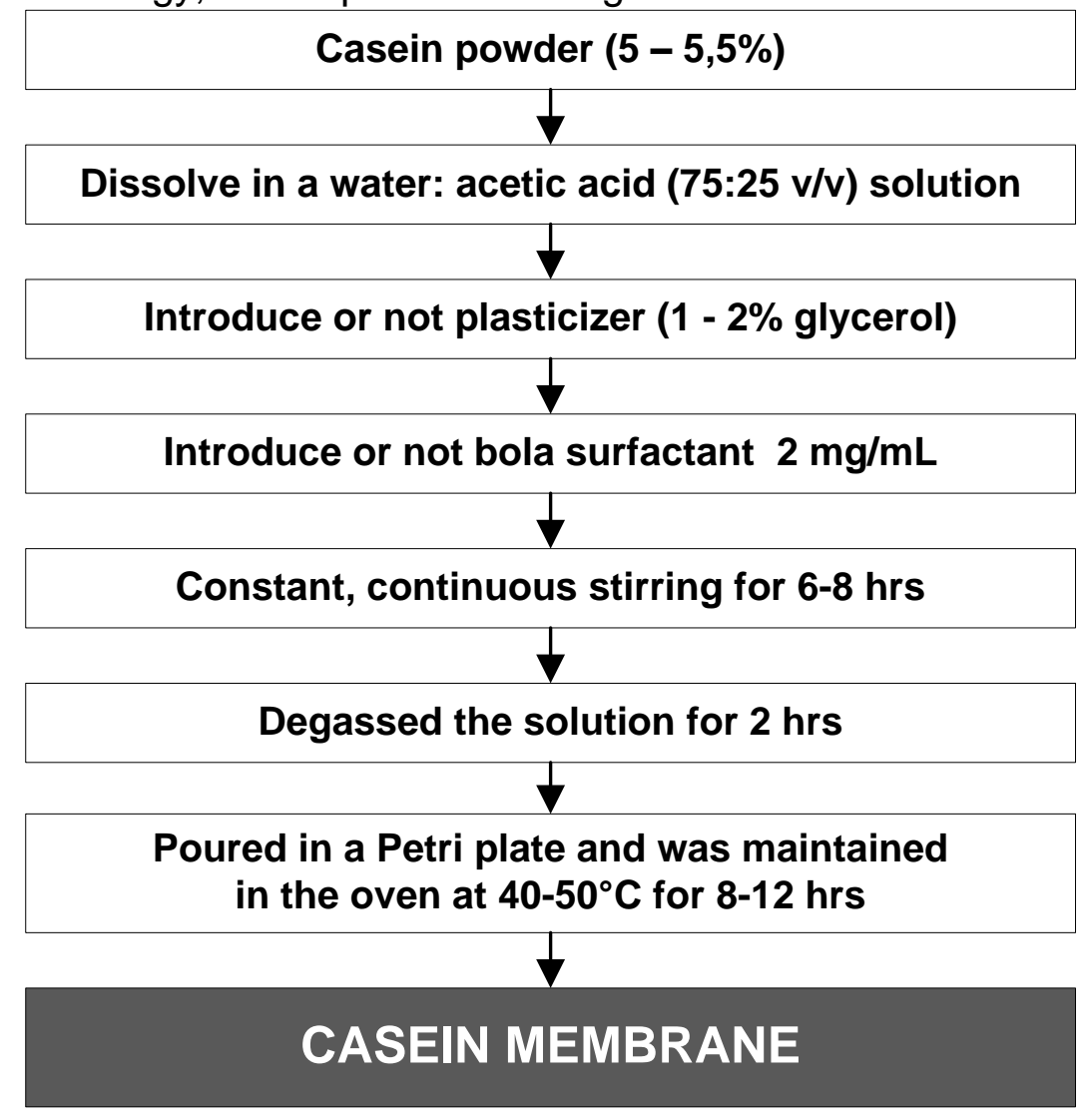

Fig. 1. Technology for obtaining casein membrane with and without plasticizer: glycerol and surfactant (classic or bola) 
These conditions allow the casein molecules from solution to be structured and to form intermolecular bonds without any cross-linking agent. Casein membranes obtained had an microporous structure, fig.2.

SEM images of the surface casein membranes gives information on the surface morphology of the unused membranes. The surface of the casein membranes consists of pores of varying size. Cross-sections of the membranes were prepared to assess internal structure. Cross-sections were cut using a scalpel and fractured. Compression of the structure is visible in Fig. 3. Casein membrane consists of a finger-like microsubstructure.

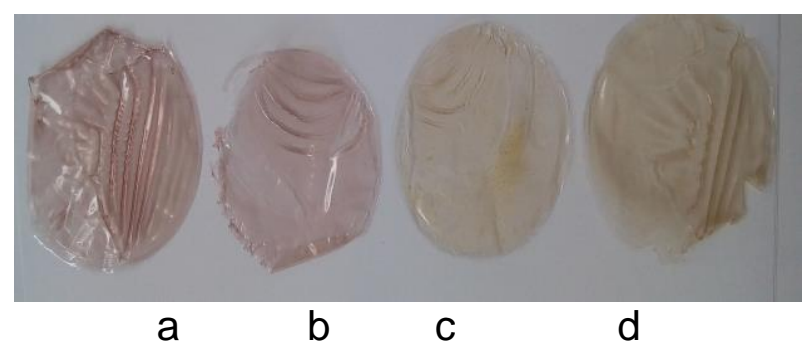

Fig.2. Images of casein membranes: acasein membrane without plasticizer and surfactant; b-casein membrane with plasticizer, c-casein membrane with plasticizer and bola surfactant, $\mathbf{d}$-casein membrane with plasticizer and classic surfactant
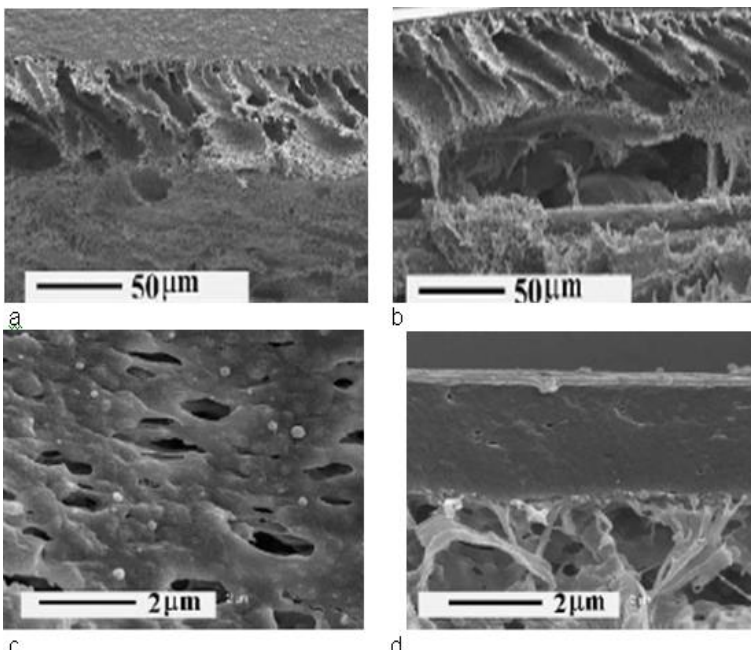

Fig.3. SEM images of cross-sections for casein membranes: a-casein membrane without plasticizer and surfactant; b-casein membrane with plasticizer, c-casein membrane with plasticizer and bola surfactant, $d$-casein membrane with plasticizer and classic surfactant

Optical microscopy of membranes confirms microstructure observed by electron microscopy. FTIR-ATR spectroscopy of casein membranes showed characteristic peaks for each type of membrane with or without plasticizer and surfactant (classic or bola).Microbiological tests were performed according to European standard using Sabouraud culture medium inoculated with Staphyloccous Aureus, placed over the samples. The strongest antibacterial effect was found for the membrane with bola surfactant.

Casein membranes interactions with surfactants-dyes and separation rates of dyes were studied with: UV-VIS, dynamic light scattering, scanning electron microscopy, contact angle. To study dyes-surfactant interactions, the following were chosen: b)casein membrane with plasticizer, c)-casein membrane with plasticizer and bola surfactant. The micellar adsorption wavelength from $470 \mathrm{~nm}$, characteristic for Orange III solution, Yellow III at $430 \mathrm{~nm}$. UV-VIS spectroscopy showed that in the absence of bola surfactant, in the plot of the normalized flux vs. the solution concentration, the Yellow III dye exhibits an extreme point at a concentration of $0.14 \mathrm{~g} / \mathrm{dL}$, both for casein membranes with or without bola surfactant. The Yellow III dye also shows a maximum 
retention for small concentrations $(0.01 \mathrm{~g} / \mathrm{dL})$. At greater concentrations, the retention on the membrane-b) is zero, respectively $40 \%$ for the membrane-c).

The maximum point can be assigned to an adsorption phenomenon on the membrane, but the membrane-c) exhibits a real retention, Fig. 4. In the case of Orange III dye the membrane-b) is fouled and hence the normalized flux is decreasing, fig.5.

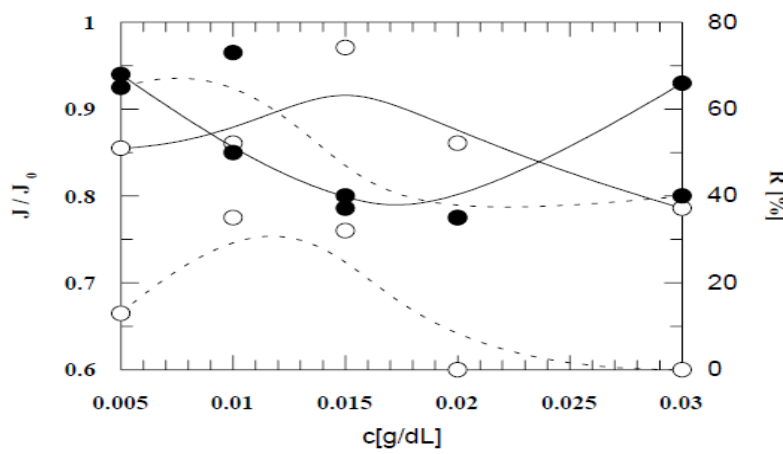

Fig.4. Dependence of the normalized flux $\left(\mathrm{J} / \mathrm{J}_{0}\right.$

- ) and retention ( $R \cdots$...) of Yellow III (Y) vs. concentration for o- membrane-b) and $\bullet$ membrane-c)

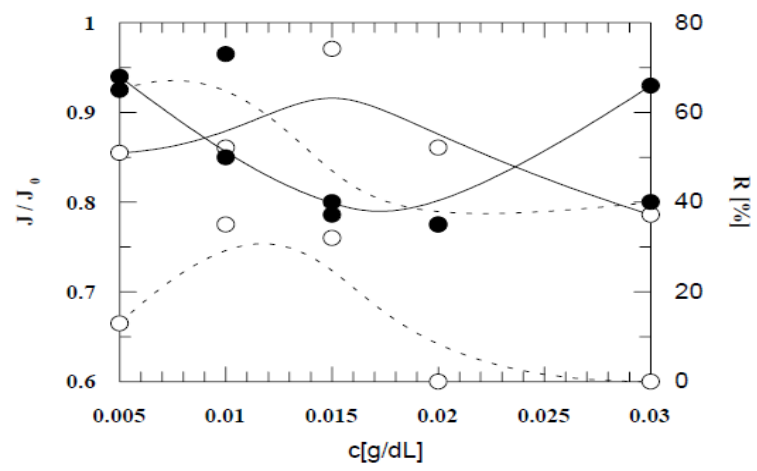

Fig.5. Dependence of the normalized flux $\left(\mathrm{J} / \mathrm{J}_{0}-\right.$ ) and retention ( $\mathrm{R} \cdots \cdot$ ) of Orange III vs. concentration for o- membrane-b) and - membrane-c)

The presence of the bola surfactant in the dye solution leads to the formation of some complexes (associates), [5]. These complexes may be swelled with an organic compound, or may be caused by the electrostatic interactions, favouring the increase of the retention both for the Orange III dye and the bola surfactant, fig.6.

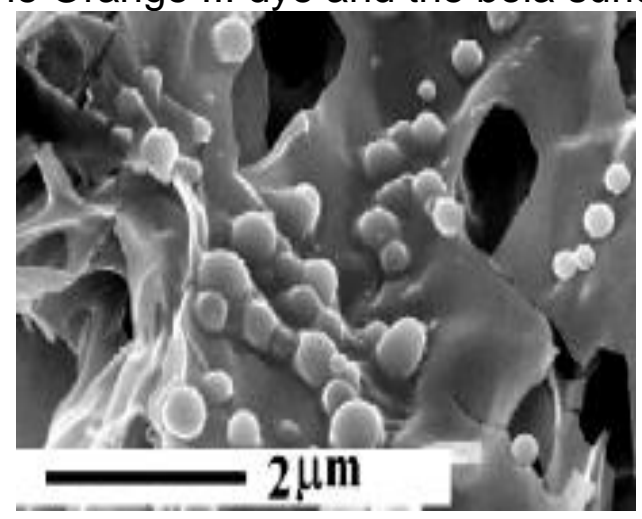

Fig.6. SEM image for associates: bola surfactant-Orange III dye on casein membrane-c), with plasticizer and bola surfactant

The membrane-c) obtained with bola surfactant exhibits lower contact angle towards water than the membrane-b) without surfactant, $120^{\circ}$ to $93^{\circ}$.

\section{Conclusions}

A variety of techniques for microstructural analysis were used to study the surface morphology and internal microstructure of casein membranes [6]. Comparison of the methods shows that either optical or SEM microscopy examination showed unused flat sheet casein membranes.

The presence of surfactants in the composition of the membranes formed was emphasized in order to maintain the hology and membrane performances. Surfactants 
in the casting solution alters the size, as well as the density of pores and the roughness of casein membranes surface. The surfactants analysed, yield membranes with small and dense pores and with smooth surface. Also, surfactants in the dyes aqueous solution influenced the separation rates.

Ecological membranes are obtained from a biodegradable biopolymer and can be used successfully in removing dyes from wastewaters. The actual European Community strategy related to maintenance of health of population, quality of life, and of the environment encourages the new technologies for pollution abatement.

\section{References}

[1] N.G. Wang, L.N. Zhang, Y.S. Lu, Y.M. Du, J. Appl. Polym. Sci. 91 (2004) 332-338.

[2] P.L.M. Barreto, A.T.N. Pires, V. Soldi, Polym. Degrad. Stab. 79 (2003) 147-152.

[3] S.S. Sohail, B.W. Wang, M.S. Biswas, J.H. Oh, J. Food Sci. 71 (2006) 255-259.

[4] D.Simion, M.Radu, F.Radu, F.Biziru, C.Alecu, A.Piscureanu, D.Vărăşteanu, E.Chican, Patent "Bolaamphiphilic material with ester structure and the process for obtaining it", No. 122542/2009, Of.de state and inventions,Part 1, No.541/08.08.2007.

[5] G.Popescu, B.G.Albu, D.Ratã, L.Moise, G.Nechifor (1994) Interfiltra'94, Intermembrane Recentes progresses in genie des procedes.

[6] Ma.Jianzhong, Fan Zhang, Giao. Yinghuan, Xu. Qunna, Zhou. Jianhua, Demetra Simion, Marius Simion, Zhang. Jing, "Surface properties of Polyacrylate/Organic Siloxane Co-modified Casein Coatings", Industrial \& Engineering Chemistry Research, 2016, in press (shenoffice@iecr.acs.org), Manuscript ID: ie-2016-03217u ( https://acs.manuscriptcentral.com/acs) 